\title{
Robust fault detection using consistency techniques for uncertainty handling
}

\author{
Esteban R. Gelso, Sandra M. Castillo, Joaquim Armengol \\ University of Girona, E17071 Girona, Spain \\ \{esteban.gelso,sandra.castillo,joaquim.armengol\}@udg.edu
}

\begin{abstract}
Often practical performance of analytical redundancy for fault detection and diagnosis is decreased by uncertainties prevailing not only in the system model, but also in the measurements. In this paper, the problem of fault detection is stated as a constraint satisfaction problem over continuous domains with a big number of variables and constraints. This problem can be solved using Modal Interval Analysis and Consistency Techniques. Consistency techniques are then shown to be particularly efficient to check the consistency of the Analytical Redundancy Relations (ARRs), dealing with uncertain measurements and parameters. Through the work presented in this paper, it can be observed that consistency techniques can be used to increase the performance of a robust fault detection tool, which is based on interval arithmetic. The proposed method is illustrated using a nonlinear dynamic model of a hydraulic system.
\end{abstract}

$\underline{\text { Keywords }}$ - Fault detection, Consistency, Uncertainty, Interval analysis.

\section{INTRODUCTION}

Uncertainty has been a common theme in a several fields. It is a term used to express that nothing is perfect in this world, at least in the sense that we perceive it.

Considering a system it is possible to identify several sources of uncertainty, even both internal and environmental sources. For instance, there is always a gap between the intrinsic properties (or conditions) and the explicit knowledge about materials and machines, for that reason perfectly identical results according to design are never produced. On the other hand, external interferences may enter the system and alter its performance and modify the output signal.

Thus, no matter how accurate the measurement is, it is only an approximation or estimate of the true value of the specific quantity subject to measurement. Never the measured valued can be totally assured. The result of a measurement should be considered complete only when accompanied with a quantitative statement of its uncertainty.

Often practical performance of several model-based fault detection and diagnosis techniques is decreased by uncertainties prevailing not only in the system model, but also in the measurements.

A fault is a malfunction in a system and may have consequences, such as economic losses derived from lower efficiency of the system or danger to people or the environment.

Different techniques have been developed in recent years that are intended to detect and diagnose faults. These techniques can be classified in different ways [1], [2]. For example, a distinction can be made between model-based techniques and techniques based on other kinds of knowledge, such as heuristic approaches, statistical approaches, learning systems, artificial neural networks, etc.

Two research communities work on model-based techniques: the FDI (Fault Detection and Isolation) community, formed by researchers with a background in control systems engineering, and the DX (Principles of Diagnosis) community, formed by researchers with a background in computer science and intelligent systems.

The collaboration between these two communities to develop more powerful tools for fault detection and diagnosis has been one of the goals of the European Network of Excellence on Model Based Systems and Qualitative Reasoning (MONET) [3], particularly of the Bridge task group.

Among the techniques developed by the FDI research community, there are classical methods, such as state observers, parity equations and parameter estimation [4], [5], [6], [7]. A method to detect faults consists in comparing the behaviour of an actual system and a model of the system. This principle is called analytical redundancy. Consider an actual system or a part of it that can be represented by a model described by the following nonlinear discrete-time equation,

$$
\boldsymbol{y}(k)=\boldsymbol{f}(\boldsymbol{y}(k-1), \ldots, \boldsymbol{y}(k-n), \boldsymbol{u}(k-1), \ldots, \boldsymbol{u}(k-m), \boldsymbol{p})
$$

where $\boldsymbol{y}(k) \in \mathbb{R}^{n_{y}} \ldots \boldsymbol{y}(k-n) \in \mathbb{R}^{n_{y}}$ are the outputs of the system at instants $k \ldots k-n, f$ is a vector of continuous 
functions, $\boldsymbol{u}(k-1) \in \mathbb{R}^{n_{u}} \ldots \boldsymbol{u}(k-m) \in \mathbb{R}^{n_{u}}$ are the inputs at instants $k-1 \ldots k-m$, and $p \in \mathbb{R}^{n_{p}}$ is a vector of parameters.

An analytical redundancy relation (ARR) is an algebraic constraint deduced from the system model which contains only measured variables. An ARR for Equation 1 is

$$
\tilde{\boldsymbol{y}}(k)=\hat{\boldsymbol{y}}(k),
$$

where $\tilde{\boldsymbol{y}}(k)$ is the measured output of the system at instant $k$ and $\hat{\boldsymbol{y}}(k)$ is an analytical output of the model at instant $k$ and computed as

$$
\hat{\boldsymbol{y}}(k):=\boldsymbol{f}(\tilde{\boldsymbol{y}}(k-1), \ldots, \tilde{\boldsymbol{y}}(k-n), \tilde{\boldsymbol{u}}(k-1), \ldots, \tilde{\boldsymbol{u}}(k-m), \boldsymbol{p})
$$

An ARR is used to check the consistency of the observations with respect to the system model. Therefore, a fault is detected when

$$
\hat{\boldsymbol{y}}(k) \neq \tilde{\boldsymbol{y}}(k)
$$

or equivalently

$$
\boldsymbol{r}(k)=\hat{\boldsymbol{y}}(k)-\tilde{\boldsymbol{y}}(k) \neq \mathbf{0},
$$

where $r$ is called the residual of the ARR.

The main problem is that the measured output $\tilde{\boldsymbol{y}}(k)$ and the computed output $\hat{\boldsymbol{y}}(k)$ are seldom the same because the model is, by definition, inaccurate, i.e. it is an approximate representation of the system. This is the consequence of the uncertainties of the system and the procedure of systems modelling.

The better model used to represent the dynamic behavior of the system, the better will be the chance of improving the reliability and performance in detection and diagnosis of faults. However, modelling errors and disturbances in complex engineering systems are unavoidable and, hence there is a need to develop robust fault diagnosis algorithms. The goal of robustness is to minimize the false and missing alarm rates due to the effects that modelling uncertainty and unknown disturbances will have on the residuals. This can be achieved in several ways, e.g. by statistical data processing, averaging, or by finding and using the most effective threshold. One way to find effective thresholds is using intervals to bound the parameters and measurements uncertainty. In this way adaptive thresholds (envelopes) could be obtained.

Some interval methods have been proposed in the context of fault detection and diagnosis, e.g. [8], [9], [10], including constraint propagation to solve fault detection problems [11], and in [12], where the problem is solved using a tool known as IntervalPeeler, based on constraint projection algorithms, 2Bconsistency, to reduce interval domains of variables without bisections.

In this paper, the uncertainties associated with the system itself and with the measurements are taken into account, also by using intervals. Through these interval techniques, another variable such a perturbation, which is unknown but bounded can also be included in the model. This variable takes into account, for example, unknown inputs, unmodeled dynamics of the actual plant or an error due to the discretization procedure.

In section II, the problem of detecting faults is stated as a Quantified Real Constraint satisfaction problem, which can be solved using the Modal Interval Analysis (MIA). The sliding time windows, which are introduced in this section, reduce the computational effort required and improve the fault detection results. This method is implemented in SQualTrack, a software package to detect faults in uncertain systems, which has been applied in the European project CHEM (Advanced Decision Support System for Chemical/Petrochemical Manufacturing Processes) [13] to detect faults in real processes.

When interval uncertainties are considered, consistency methods which combine interval methods and constraint satisfaction techniques can be used to solve different problems. Constraint satisfaction techniques implement local reasoning on constraints to remove inconsistent values from variable domains. In practice, the set of inconsistent values is computed by means of interval reasoning. In section III the alternative to use local and global consistency techniques such as Hull and Box consistency, is explored. The fault detection problem is shown like a constraint satisfaction problem and the resolution of this problem is performed by the solver RealPaver [14].

The aim of this paper is to show the usefulness of the consistency methods to solve fault detection problems. The application of approaches of consistency (using classical and modal intervals) is illustrated with a hydraulic plant example and the obtained results are compared. Some fault detection results are presented in section IV. Finally, section V provides some conclusions and outlines the future work.

\section{RoBUST FAULT DETECTION USING INTERVALS}

The model based fault detection using modal intervals, is based on the calculation of effective thresholds (adaptive thresholds or envelopes) to bound the uncertainty of parameters and measurements. To compute the envelope limits, it is necessary to compute the range of a function in a given parameter space at each prediction step, which is very costly. SQualTrack [15] calculates iteratively external estimates

$$
\hat{Y}_{e x}(k) \supseteq \hat{Y}(k)
$$

of the predicted behavior from the model $\hat{Y}(k)$, so the cost is heavily reduced. These estimates are closer at each iteration and, after an infinite number of iterations, the exact range $\hat{Y}(k)$ would be calculated, but the algorithm stops when the external estimate is close enough to detect the fault, thus saving much computational effort for the detection of faults. However, if no fault is detected, the algorithm will never stop. This drawback can be overcome by using an internal estimate,

$$
\hat{Y}_{i n}(k) \subseteq \hat{Y}(k)
$$

which is included in the exact envelope. If the measurement is within this envelope, then the fault, if it exists, will not be 
detected, and so the algorithm will stop iterating. The internal and external estimates of the exact envelope, which are depicted in Fig. 1, define three zones.

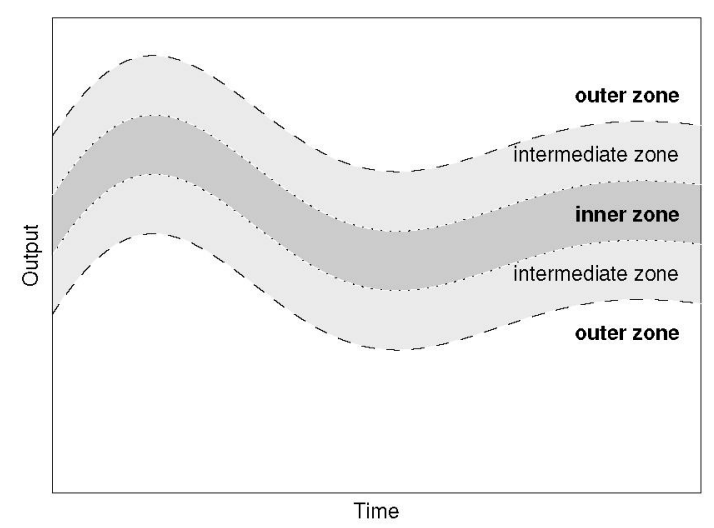

Fig. 1. THE THREE ZONES DEFINED BY THE INTERNAL AND EXTERNAL ESTIMATES OF THE EXACT ENVELOPE.

Therefore a fault is detected when the measured value is either larger or smaller than the predicted value by the external estimate or in other words, when the output of the model is not consistent with the measured output. This assertion is expressed through the logical statement,

$$
\begin{aligned}
& \neg((\exists \tilde{\boldsymbol{y}}(k) \in \tilde{\boldsymbol{Y}}(k))(\exists \hat{\boldsymbol{y}}(k) \in \hat{\boldsymbol{Y}}(k)) \hat{\boldsymbol{y}}(k)-\tilde{\boldsymbol{y}}(k)=\mathbf{0}) \Leftrightarrow \\
& (\forall \tilde{\boldsymbol{y}}(k) \in \tilde{\boldsymbol{Y}}(k))(\forall \hat{\boldsymbol{y}}(k) \in \hat{\boldsymbol{Y}}(k)) \boldsymbol{r}(k) \neq \mathbf{0} .
\end{aligned}
$$

The use of the Modal Interval Analysis [16] in the fault detection system guarantees that a fault exists when the measurement is out of the external envelope (in the outer zone), so this method does not generate false alarms. If there were false alarms, they would indicate that either the interval model does not represent the system adequately, or that the interval measurements do not represent the true values of the variables. On the other side, if the measurement is in the intermediate zone or in the inner zone there can be missed alarms.

Actually, the consistency between the interval model and the real process is performed using interval measurements, which are obtained from the measurements taking into account the uncertainties (noise, bias...) of the sensors.

Any measurement belonging to a past time point can be used as initial state to compute the envelopes at the current time point. The time interval from this initial time point to the current one is called time window. If the window used at each prediction step has always the same length, then a sliding time window is being considered [17].

Then, for a window of length $w$, in accordance with Equation 8 , a system is faulty if

$(\forall \tilde{\boldsymbol{y}}(k) \in \tilde{\boldsymbol{Y}}(k))(\forall \hat{\boldsymbol{y}}(k) \in \hat{\boldsymbol{Y}}(k \mid k-w)) \hat{\boldsymbol{y}}(k \mid k-w)-\tilde{\boldsymbol{y}}(k) \neq 0$,

where,

$$
\hat{\boldsymbol{y}}(k \mid k-w)=\boldsymbol{f}(\tilde{\boldsymbol{y}}(k-w), \tilde{\boldsymbol{u}}(k-1), \ldots, \tilde{\boldsymbol{u}}(k-w), \boldsymbol{p})
$$

The number of missed alarms is reduced by using several window lengths simultaneously, as a fault is detected when there is an inconsistency in a time window. Therefore, this method maximizes the detection of faults and, at the same time, minimizes the number of computations required.

The iterative computation of the external and the internal estimations of the exact envelope is made by a branch-and-bound algorithm which is very efficient because it uses the Modal Interval Analysis. As a conspicuous property, they eliminate larger subspaces more quickly than other techniques [18]. In this way, results of fault detection with quite less computation mass and without false alarm rate have been obtained.

\section{FAULT DETECTION AS A CONSTRAINT SATISFACTION PROBLEM}

Many engineering problems can be formulated in a logical form by means of some kind of first order predicate formulas: formulas with the logical quantifiers, universal and existential, a set of real continuous functions, equalities and inequalities and variables ranging over real interval domains.

As defined in [19], a numerical constraint satisfaction problem is a triple $\mathcal{C S P}=(\mathcal{V}, \mathcal{D}, \mathcal{C}(x))$ defined by

1. a set of numeric variables $\mathcal{V}=\left\{x_{1}, \ldots, x_{n}\right\}$,

2. a set of domains $\mathcal{D}=\left\{D_{1}, \ldots, D_{n}\right\}$ where $D_{i}$, a set of numeric values, is the domain associated with the variable $x_{i}$,

3. a set of constraints $\mathcal{C}(x)=\left\{C_{1}(x), \ldots, C_{m}(x)\right\}$ where a constraint $C_{i}(x)$ is determined by a numeric relation (equation, inequality, inclusion, etc.) linking a set of variables under consideration.

The fault detection problem can be represented by a constraint satisfaction problem where the set of variables is

$$
\begin{aligned}
\mathcal{V}= & \left\{p_{1}, \ldots, p_{n_{p}}, \tilde{y}(k-w-n), \ldots, \tilde{y}(k),\right. \\
& \tilde{u}_{1}(k-w-m), \ldots, \tilde{u}_{1}(k-1), \ldots, \\
& \left.\tilde{u}_{n_{u}}(k-w-m), \ldots, \tilde{u}_{n_{u}}(k-1)\right\}
\end{aligned}
$$

the set of domains is

$$
\begin{aligned}
\mathcal{D}= & \left\{P_{1}, \ldots, P_{n_{p}}, \tilde{Y}(k-w-n), \ldots, \tilde{Y}(k),\right. \\
& \tilde{U}_{1}(k-w-m), \ldots, \tilde{U}_{1}(k-1), \ldots, \\
& \left.\tilde{U}_{n_{u}}(k-w-m), \ldots, \tilde{U}_{n_{u}}(k-1)\right\}
\end{aligned}
$$

and the set of $w$ constraints is

$$
\begin{aligned}
\mathcal{C}= & \{f(\tilde{y}(k-w), \ldots, \tilde{y}(k-w-n), \tilde{\mathbf{u}}(k-w), \ldots, \tilde{\mathbf{u}}(k-w-m), \mathbf{p})- \\
& -\tilde{y}(k-w+1)=0, \ldots, \\
& f(\tilde{y}(k-1), \ldots, \tilde{y}(k-n), \tilde{\mathbf{u}}(k-1), \ldots, \tilde{\mathbf{u}}(k-m), \mathbf{p})-\tilde{y}(k)=0\} .
\end{aligned}
$$

Consistency techniques can be used to contract the domains of the variables involved [20], [21], [22] removing inconsistent values. In particular for the fault detection application, they are used to guarantee that the model of a residual is inconsistent when there is no solution. The algorithms that are based 
on consistency techniques are actually "branch and prune" algorithms, i.e., algorithms that can be defined as an iteration of two steps [20]:

1. Pruning the search space by reducing the intervals associated with the variables until a given consistency property is satisfied.

2. Generating subproblems by splitting the domains of a variable

Most interval constraint solvers are based on either hullconsistency (also called 2B-consistency) or box-consistency (or a variation of these ones) [21]. Box-consistency tackles the problem of hull-consistency for variables with many ocurrences in a constraint. The aforementioned techniques are said to be local: each reduction is applied over one domain with respect to one constraint. Better pruning of the variable domains may be achieved if complementary to a local property, some global properties are also enforced on the overall constraint set.

In this paper, a solution of the fault detection CSP is performed by the solver RealPaver [14]. Algorithm BC4 (a combination of hull consistency and box consistency), and a strong technique, called weak-3B consistency, are used in Section IV.

\section{APPLICATION EXAMPLE: TWO COUPLED WATER TANKS}

\section{A. System description}

A well-known dynamical example of a system based on two coupled water tanks (as in [10] but with nonlinear equations) will be used to explain the obtainment of additional information from the model for diagnostic reasoning. Fig. 2 shows a schematic drawing of the system.

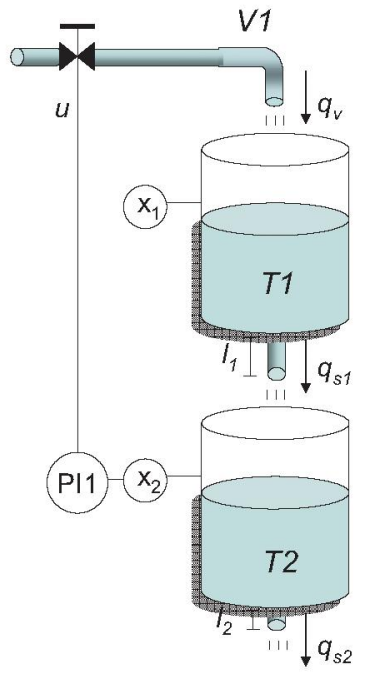

Fig. 2. Diagram OF THE COUPLED WATER TANKS SYSTEM.

The system is composed of two tanks, $T 1$ and $T 2$, a valve, $V 1$, and a controller, $P I 1$, which receives the current level of
$T 2$ as the input, and controls a valve, $V 1$, which regulates the flow of water to $T 1$.

\section{B. Model equations}

The system is described by the elementary analytical relations (EAR) shown in Table I.

TABLE I.

ELEMENTARY ANALYTICAL RELATIONS OF THE TWO COUPLED TANKS SYSTEM

\begin{tabular}{|l|l|l|}
\hline & Elementary Relations & Component \\
\hline (a) & $q_{v}=k u^{3}$ & Valve \\
(b) & $S_{1} \frac{d x_{1}}{d t}=q_{v}-q_{s 1}$ & Upper tank \\
(c) & $q_{s 1}=k_{s 1} \sqrt{x_{1}+l_{1}}$ & Output pipe upper tank \\
(d) & $S_{2} \frac{d x_{2}}{d t}=q_{s 1}-q_{s 2}$ & Lower tank \\
(e) & $q_{s 2}=k_{s 2} \sqrt{x_{2}+l_{2}}$ & Output pipe lower tank \\
(f) & $\tilde{u}=u$ & D/A converter \\
(g) & $\tilde{x}_{1}=x_{1}$ & $x_{1}$ sensor \\
(h) & $\tilde{x}_{2}=x_{2}$ & $x_{2}$ sensor \\
\hline
\end{tabular}

The terms $q_{v}, q_{s 1}$ and $q_{s 2}$ denote the volumetric flows, $x_{1}$ and $x_{2}$ are the heights of the water in tanks $T 1$ and $T 2$, respectively, and $u$ is the output signal of the controller. The variables $u$, $q_{v}, q_{s 1}, q_{s 2}, x_{1}$, and $x_{2}$ are unknown, $\tilde{u}, \tilde{x_{1}}$, and $\tilde{x_{2}}$ are known variables obtained from sensors, and $k, k_{s 1}, k_{s 2}, S_{1}, S_{2}, l_{1}$ and $l_{2}$ are the constant parameters of the system.

All the variables and parameters are considered as intervals for the consistency test using SQualTrack and RealPaver. The intervals for the measurements include the accuracy error and the noise level, and for the parameters, they were estimated using a strong consistency technique with data in a fault free scenario.

The faults considered, are sensor faults, actuator faults and process faults (leakages and clogging in the output pipes of $T 1$ and $T 2$ ).

\section{Consistency-based diagnosis}

Two analytical redundancy relations were obtained with the structural analysis, which are minimal with respect to the set of constraints used in the model, (11) and (12).

$$
\begin{aligned}
r_{1}= & \tilde{x_{1}}(k)-\tilde{x_{1}}(k-1)+\frac{T_{s}}{S_{1}}\left(-k \tilde{u}(k-1)^{3}+k_{s 1} \sqrt{\tilde{x_{1}}(k-1)+l_{1}}\right) \\
r_{2}= & \tilde{x_{2}}(k)-\tilde{x_{2}}(k-1)+ \\
& +\frac{T_{s}}{S_{2}}\left(-k_{s 1} \sqrt{\tilde{x_{1}}(k-1)+l_{1}}+k_{s 2} \sqrt{\tilde{x_{2}}(k-1)+l_{2}}\right)
\end{aligned}
$$



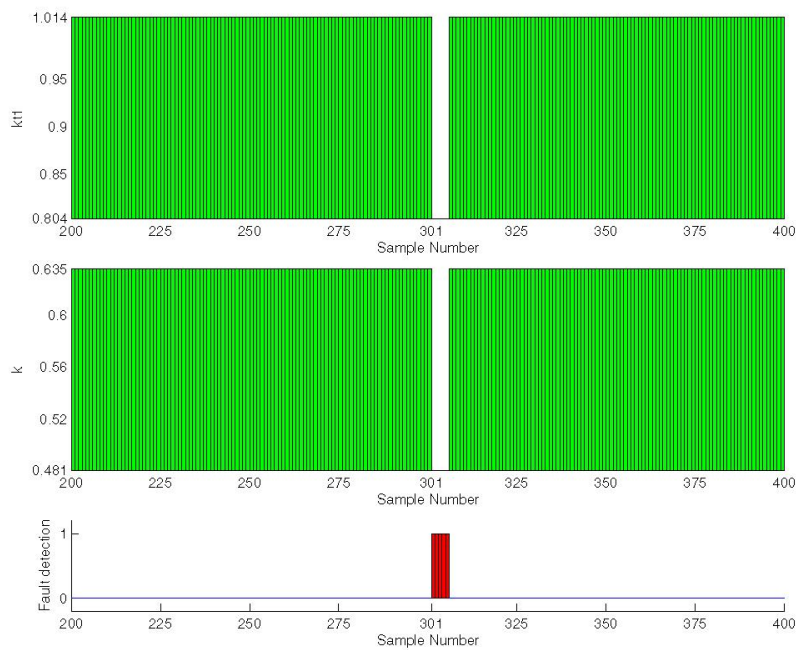

Fig. 3. WEAK-3B CONSISTENCY FAULT DETECTION USING $r_{1}$ CORRESPONDING TO A FAULT IN THE SENSOR $x_{1}$ BEGINNING AT SAMPLE 300. The FAUlT IS DETECTED FROM SAMPLE 301.
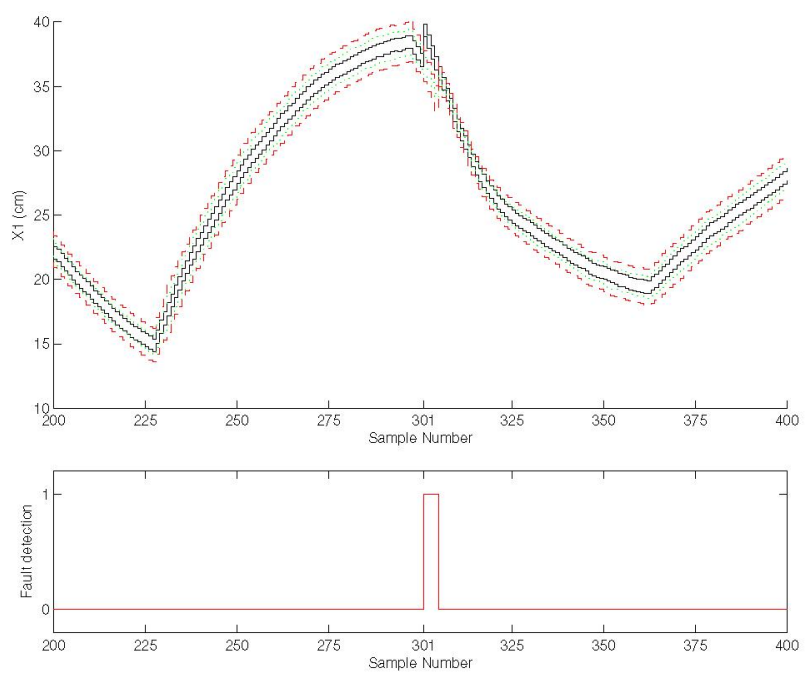

Fig. 4. SQuALTRACK FAUlt Detection using $r_{1}$ CORRESPONDING TO A FAULT IN THE SENSOR $x_{1}$ BEGINNING AT SAMPLE 300. THE FAULT IS DETECTED FROM SAMPLE 301.

\section{Simulation results}

In this section two faulty scenarios are considered, (i) a fault (bias) in the level sensor $x_{1}$, and (ii), a leakage fault in the tank $T 1$. The fault detection results obtained by using the BC4 algorithm and Weak-3B consistency technique, are similar. So afterwards, SQualTrack and Weak-3B consistency technique are compared.

In Fig. 3 and 4, obtained results in the case of bias are shown. The fault in the sensor begins at sample 300 and is detected at sample 301 using a window length 5 . The fault detection alarm is turned on by both the Weak-3B consistency technique and SQualTrack in a simultaneous way. Fig. 3 shows that, there is
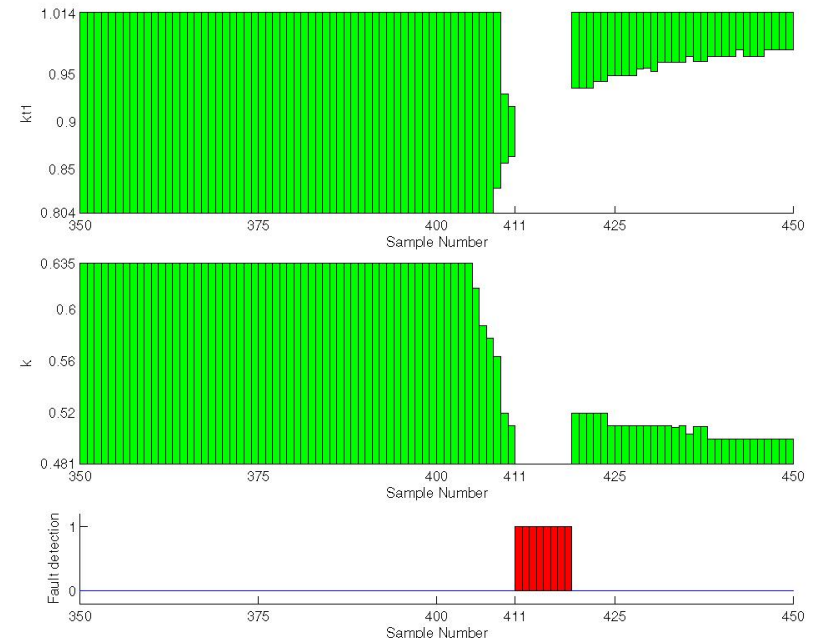

Fig. 5. WEAK-3B CONSISTENCY FAULT DETECTION USING $r_{1}$ CORRESPONDING TO A LEAKAGE IN $T_{1}$ BEGINNING AT SAMPLE 400 . The FAULT IS DETECTED FROM SAMPLE 411.
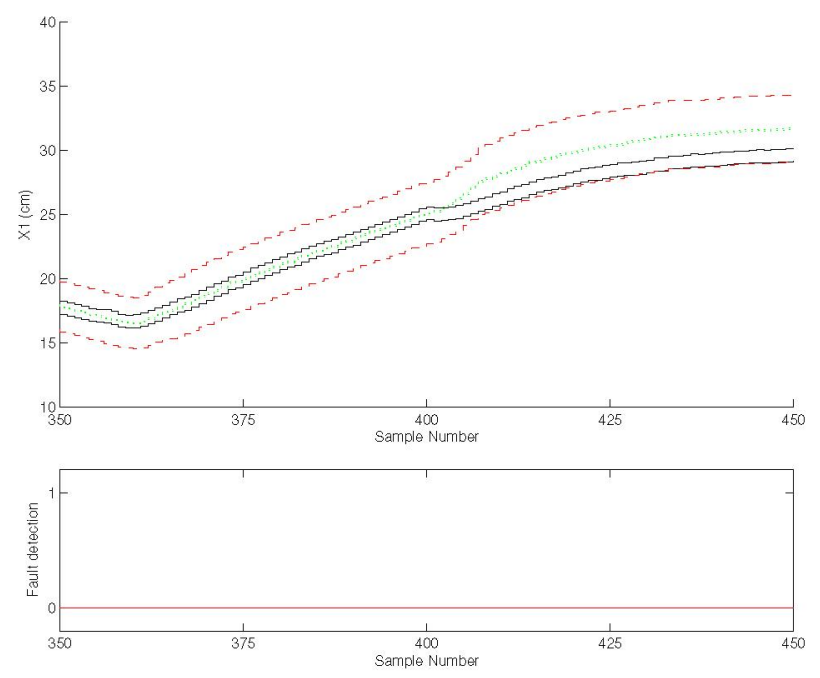

Fig. 6. SQUALTRACK FAULT DETECTION USING $r_{1}$ CORRESPONDING TO A LEAKAGE IN $T_{1}$ BEGINNING AT SAMPLE 400 . The FAULT IS NOT DETECTED.

not a consistent region of parameters when the fault is detected. Fig. 4 shows the output envelope measured (solid black line) is out of the external envelope calculated (dashed red line) when the fault is detected. The internal envelope calculated, is the green dotted line.

In Fig. 5 and 6, obtained results in the case of leakage are shown. The leakage begins at sample 400 and is detected at sample 411 using a window length 25 . The fault detection alarm is turned on by the Weak-3B consistency technique. Fig. 5 shows how the consistency region of parameters starts to disappear when the fault occurs. 


\section{CONCLUSION AND FUTURE WORK}

In order to represent parameter and measurement uncertainties explicitly, a special case presented in this paper, is when they can be handled by means of interval models in which, the true value of the specific quantity is allowed to vary within numeric intervals.

When interval uncertainties are considered, consistency methods can be used to solve different problems. In this paper the problem is presented as a constraint satisfaction problem over continuous domains with a big number of variables and constraints. Through the obtained results, consistency techniques are shown to be particularly efficient to check the consistency of the Analytical Redundancy Relations (ARRs), dealing with uncertain measurements and parameters. These techniques became a new tool to check the consistency of residuals described by discrete-time state equations. Also, it could be said that consistency techniques could be used for solving the fault detection problem when the system is described using a space-state model or, even, when the system is described by several equations. In the future, the compatibility between consistency techniques and SQualTrack fault detection tests must be studied, in order to increase the performance of this robust fault detection tool.

\section{ACKNOWLEDGMENT}

This work has been partially funded by the European Regional Development Fund and European Social Fund of the European Union, the Spanish Government (Plan Nacional de Investigación Científica, Desarrollo e Innovación Tecnológica, Ministerio de Ciencia y Tecnología) through the coordinated research projects grant No. DPI2003-07146-C02-02 and grant No. DPI2005-08668-C03-02, by the grant No. 2005SGR00296 and the Departament d'Innovació, Universitats, i Empresa of the Government of Catalonia.

\section{REFERENCES}

[1] K. Balakrishnan and V. Honavar, "Intelligent diagnosis systems," Journal of Intelligent Systems, vol. 8, no. 3, pp. 239-290, 1998.

[2] V. Venkatasubramanian, R. Rengaswamy, K. Yin, and S.N. Kavuri, "A review of process fault detection and diagnosis: Part I: Quantitative model-based methods," Computers and Chemical Engineering, vol. 27, pp. 293-311, 2003.

[3] MONET, "European network of excellence on model based systems and qualitative reasoning," http://monet.aber.ac.uk: 8080/monet/index.htmi, 1998
[4] M. Blanke, M. Kinnaert, J. Lunze, and M. Staroswiecki, Diagnosis and Fault-Tolerant Control, Springer, 2003.

[5] J. Chen and R.J. Patton, Robust model-based fault diagnosis for dynamic systems, Kluwer, 1998.

[6] J. J. Gertler, Fault Detection and Diagnosis in Engineering Systems, Marcel Dekker, 1998.

[7] R. J. Patton, P. M. Frank, and R. N. Clark, Issues of fault diagnosis for dynamic systems, Springer, 2000.

[8] J. Armengol, J. Vehí, L. Travé-Massuyès, and M. Á. Sainz, "Interval model-based fault detection using multiple sliding time windows," in 4th IFAC Symposium on Fault Detection, Supervision and Safety for Technical Processes SAFEPROCESS 2000. Budapest. Hungary, 2000, pp. 168-173.

[9] V. Puig, J. Quevedo, and T. Escobet, "Model-based fault diagnosis of dynamic processes: comparing FDI and DX," in IV Jornadas de ARCA: Sistemas Cualitativos y Diagnosis, 2002, p. 91.

[10] Stéphane Ploix and Cyril Follot, "Fault diagnosis reasoning for set-membership approaches and application," in IEEE International Symposium on Intelligent Control, 2001.

[11] A. Stancu, V. Puig, and J. Quevedo, "Gas turbine model-based robust

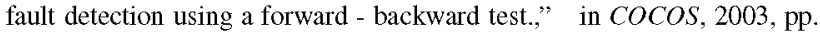
$154-170$.

[12] V. Puig, C. Ocampo-Martínez, S. Tornil, and A Ingimundarson, "Robust fault detection using set-membership estimation and constraints satisfaction," in 17th International Workshop on Principles of Diagnosis DX, 2006, pp. 227-234.

[13] CHEM Consortium, "Advanced decision support system for chemical/petrochemical manufacturing processes," http://cordis.europa.eu/search/index.cfm?fuseacti on=proj . simpledocument $\&$ PJ_RCN $=5074917 \& \mathrm{CFID}=6489798$ CE TOKEN $=20166382,2000$.

[14] L. Granvilliers and F. Benhamou, "Aloorithm 852: Realpaver: an interval solver using constraint satisfaction techniques." ACM Trans. Math. Softw., vol. 32, no. 1, pp. 138-156, 2006.

[15] J. Armengol, J. Vehí, M. Á. Sainz, and P. Herrero, "Fault detection in a pilot plant using interval models and multiple sliding time windows," in 5th IFAC Symposium on Fault Detection, Supervision and Safety for Technical Processes SAFEPROCESS 2003. Washington, D.C., U.S.A., 2003.

[16] SIGLA/X, Applications of Interval Analysis to Systems and Control. Proceedings of MISC 1999, chapter Modal Intervals, pp. 157-227, Universitat de Girona, 1999.

[17] J. Armengol, J. Vehí, L. Travé-Massuyès, and M. Á. Sainz, "Application of multiple sliding time windows to fault detection based on interval models," in 12th International Workshop on Principles of Diagnosis DX 2001. San Sicario, Italy, Sh. Mcllraith and D. Theseider Dupré, Eds., 2001, pp. 9-16.

[18] J. Armengol, J. Vehí, L. Travé-Massuyès, and M. Á. Sainz, "Application of modal intervals to the generation of error-bounded envelopes," Reliable Computing, vol. 7, no. 2, pp. 171-185, April 2001.

[19] Sergey Petrovich Shary, "A new technique in systems analysis under interval uncertainty and ambiguity," Reliable Computing, vol. 8, pp. $321-418,2002$.

[20] H. Collavizza, F. Delobel, and M. Rueher, "Comparing partial consistencies," Reliable Computing, vol. 5, pp. 213-228, 1999.

[21] F. Benhamou, F. Goualard, L. Granvilliers, and J. F. Puget, "Revising hull and box consistency," in Proceedings of the International Conference on Logic Programming, Las Cruces, NM. 1999, pp. 230-244.

[22] J. Cruz and P. Barahona, "Maintaining global hull consistency with local search for continuous CSPs.," in COCOS, 2002, pp. 178-193. 